\title{
Evaluate the Effect of Duration for Injury on International Knee Documentation Committee (IKDC) Score With an Anterior Cruciate Ligament Injury Patients
}

\author{
Mona H. Ahmed ${ }^{1}$, Zienab A. Mohamad ${ }^{2}$, Hatem G. Said ${ }^{3}$ \& Sahar A. Abd-El mohsen ${ }^{4}$. \\ M.D. Nursing (Students' Hospital), Assiut University, Egypt. \\ Professor of Medical Surgical Nursing, Faculty of Nursing, Assiut University, Egypt. \\ Professor of orthopedic Surgery, Faculty of Medicine, Assiut University, Egypt. \\ Lecturer of medical-surgical Nursing, Faculty of Nursing, Assiut University.
}

\begin{abstract}
The anterior cruciate ligament (ACL) tear is one of the most common knee injuries. Aim; to evaluate the effect of duration for injury on IKDC score with an anterior cruciate ligament injury patients. Patients and methods; Nonequivalent control group design was utilized to conduct this study. The study sample, 60 adult patients with an ACL injury, patients were divided into two equal groups, group one their injury less than 3 months and group two their duration of injury more than 3 months. Tools; the International Knee Documentation Committee (IKDC) scale and exercise program. Results, the mean age were (24.7), and the majority of them were students, regarding the IKDC scale there was a highly statistically significant difference between both groups during pre-operative and all of the follow up periods. Conclusion; An improvement was observed at $3 \& 6$ months follow up of patients with an ACL injury of less than 3 months than those patients whose injury more than 3 months. Recommendation; providing patients with instructions for seeking medical advice for their sports injuries of great importance for early diagnosis and management of their injuries.
\end{abstract}

\section{Keywords: Anterior Cruciate Ligament Injury, Duration Of Injury\& IKDC Score.}

\section{Introduction}

The anterior cruciate ligament (ACL) is critical for knee role in athletes who require knee constancy in activities such as running, cutting, jumping, and kicking. The ACL originates on the posteromedial aspect of the lateral femoral condyle and inserts anterior to and between the intercondylar distinctions of the tibia. The ACL functions as the primary restraint to limit anterior tibial translation and as a secondary limit to internal rotation of the tibia (Aglietti, et al., 2014).

Diagnosis of an ACL injury depends on: first history; the injury often is associated with immediate pain and an audible "pop.", swelling of the knee is noted within a few hours (acute hemarthrosis). The patient often states that the knee feels too unstable to continue playing and that weight bearing is difficult. Physical exam; a careful physical examination can diagnose most ACL injuries (Dugan, 2015).

Treatment: Initial stabilization; the initial treatment of the acutely injured ACL is splinting and the use of crutches for comfort and early active range of motion (ROM). The goal is to obtain full ROM. Ice, elevation, and analgesics are prescribed in the initial post-injury period. Treatment decisions should be based on many factors, including patient age, activity level, type of sporting activity (especially jumping, cutting, and pivoting sports), degree of instability, and associated knee pathology. Treatment options include; physical therapy, medications and surgery (Shea, 2015).
Rehabilitation is a problem-solving and educational process aimed at reducing the disability experienced by someone (with physical impairment) as a result of a disease, but always within the limitations imposed by available resources and by the underlying disease (Baum \& Irrgang 2013).

Nurses play a vital role in rehabilitation for patients who had ACL reconstruction surgery. Rehabilitation therapy will be needed, rehabilitation will include the use of crutches and, possibly, a knee brace range-ofmotion exercises to regain full knee motion, musclestrengthening and stability exercises. The nurse gives teaching about weight bearing limit, exercise restraints. Nurses' Initial aims are to reduce pain and swelling in knee, regain normal joint movement and strengthen the muscles around the knee and assess for signs of complications (Sharonl, et al., 2007).

\section{Significance of the study}

A delay in the timing of surgery and rehabilitation of $>3$ months compared with performing the surgery and rehabilitation $\leq 3$ months after the anterior cruciate ligament (ACL) injury leads to an increased risk of injuries or degenerative changes in the ACLdeficient knee. According to Assiut University hospital records 350 patients underwent ACL surgery in 2017. 


\section{Aim of the study}

This study aim was to evaluate the effect of duration for injury on IKDC score with an anterior cruciate ligament injury patient.

\section{Research question}

Does the duration of an anterior cruciate ligament injury effects on the IKDC score?

\section{Patients and Methods}

Research design; Non-equivalent control group design was utilized in this study; the sample is common in the same type of injury but not in the same sport

Setting: this study was conducted in the department of orthopedic surgery and out-patient arthroscopy clinic at Assiut University hospital.

Patients; A sample of 60 adult patients with an ACL injury was included in the study, both gender, having an ACL injury; their age ranges from 18 to 60 years, patients were taken from time of admission in the department (pre operatively) and 3 months, 6 months and 12 months (post operatively) follow up in the out-patient arthroscopy clinic, patients were divided into two equal groups (30 patients for each), group one their injury less than 3 months and group two their duration of injury more than 3 months.

\section{Exclusion Criteria}

1. Patients with radiographic evidence of osteoarthritis (grade 3 or 4 osteoarthritis according to the Kellegren and Lawrence system) or inflammatory arthritis at the time of injury or first-time clinical evaluation .

2. Patients who sustained intra-cerebral/spinal cord injury at the time of initial presentation.

\section{Sample size calculatio:}

$\mathrm{G}$ power program 3.1.3 was used to calculate sample size in order to detect a-significant difference in mean of (IKDC) between two groups under the study hypothized; effect size 0.7 , power $80 \%, \infty$ error 0.05 , allocation ratio $1: 1$ and 26 patients were included in each group with total sample size 52 patients.

- Sample size was increased 4 patients in each group.

- 80 patients were included in this study, 20 dropouts because some patients were not living in Assiut city and it was difficult for them to come and some of them were not cooperative with the study, the final sample size was estimated on 60 patients.

Tools; Two tools were used in this study and were developed by the researcher to collect the necessary data for this study.

Tool I: Subjective knee evaluation using International Knee Documentation Committee (IKDC) scale (Anderson, et al., 2000) it was divided into two parts:

Part 1: Demographic patient characteristics: which were consist of: patient's name, age, gender, level of education, occupation, time of ACL injury, and surgery day.

Part 2: IKDC subjective knee evaluation form: This questionnaire has been designed to give information as to how patient's knee is able to do the usual activities .

This scale includes 3 sections:

1. Symptoms

2. Sports activity

3. Function and activity of daily living.

Scoring syste :

$$
\text { IKDC Score }=\left[\frac{\text { Sum of Items }}{\text { Maximum Possible Score }}\right] \times 100
$$

Tool II: Exercise program for patients with an ACL injury.

This tool was developed to teach the patients the rehabilitation exercises which were given in a simplified Arabic booklet to the patients and then those patients were followed up to assess the effect of the rehabilitation on their condition comparatively with their injury duration, and to see which group would have a better functional level, it consists of 5 phases: week 1-2 after the operation, week 2-6 after the operation, weeks 6-12 after the operation, preparation for return to sport 3-6 months, and return to sport at 6-9 months.

Methods

1. Content validity was established by panel of 5 expertises from medical and surgical nursing staff who reviewed the tools for clarity, relevance, comprehensiveness, understanding, applicability and easiness, minor modifications were required.

2. Content reliability was estimated by Alpha Cronbach's test and its result was $\mathrm{R}=0.68$.

3. A pilot study was carried out in December 2016 to test the feasibility and practicability of the study tools on $10 \%$ (6 patients) of the sample they were involved to the study. It also provided an estimate of time needed to fill out the tools.

4. Data were collected from orthopedic surgery and out-patient arthroscopy clinic at Assiut University hospital during the period from December /2016 to April /2018. The tools were filled out through interviewing each patient individually.

\section{Ethical consideration}

-there was no risk for patients participating in the study, patients was assured confidentiality and anonymity and their right to withdraw from the study without any rational at any time during the study after attaining their informed consent for participation in the study.

\section{Statistical analysis}

Data collected were analyzed by computer program SPSS" version.17" Chicago. USA. Data expressed as 
"mean $\square$ standard deviation" "number, percentage". Using $\mathrm{T}$ test to determine significance for numeric variables. Using Chi. square test to determine significance for non-parametric variables. Using Pearson correlation for numeric variables in the same group.

n.s $\mathrm{P}>0.05$ no significance.
$* * \mathrm{P}<0.01$ moderate significance.

$* * * \mathrm{P}<0.001$ highly significance.

Limitation of the study

It was difficult to follow up some of patients because they were not living in Assiut city and it was difficult for them to come, some of patients were not cooperative with the study.

\section{Results}

Table (1): Frequency distribution of patients according to their Socio-demographic characteristics.

\begin{tabular}{|c|c|c|c|c|c|}
\hline \multirow{2}{*}{ Personal data } & \multicolumn{2}{|c|}{$\begin{array}{c}\text { ACL injury }<3 \text { months } \\
\mathrm{N}=30\end{array}$} & \multicolumn{2}{|c|}{$\begin{array}{c}\text { ACL injury > } 3 \text { months } \\
N=30\end{array}$} & \multirow{2}{*}{$\begin{array}{l}\begin{array}{c}\text { Total } \\
\mathrm{N}=60\end{array} \\
\text { N. } \%\end{array}$} \\
\hline & $\mathbf{N}$. & $\%$ & N. & $\%$ & \\
\hline $\begin{array}{l}\text { Age group } \\
18 y r s \text { to } 60 y r s\end{array}$ & \multirow{2}{*}{\multicolumn{5}{|c|}{$\underline{\text { Means } \pm \text { SD }}$}} \\
\hline Level of education & & & & & \\
\hline Secondary school & 9 & 30 & 9 & 30 & $18(30.0)$ \\
\hline University & 21 & 70 & 21 & 70 & $42(70.0)$ \\
\hline \multicolumn{6}{|l|}{ Occupation } \\
\hline Student & 15 & 50 & 22 & 36.7 & $37(61.7)$ \\
\hline Worker & 10 & 33.3 & 5 & 8.3 & $15(25.0)$ \\
\hline Private & 2 & 6.7 & 2 & 3.3 & $4(6.7)$ \\
\hline Farmer & 3 & 10 & 1 & 1.7 & $4(6.7)$ \\
\hline
\end{tabular}

Table (2): Comparison between both groups using the International Knee Documentation Committee (IKDC) scale; Symptoms.

\begin{tabular}{|c|c|c|c|c|c|}
\hline Symptoms & Group case & N. & Mean & Std. Deviation & P.V. \\
\hline Pre- operative & $\begin{array}{l}\text { ACL injury < 3 months } \\
\text { ACL injury > 3 months }\end{array}$ & 60 & 27.9167 & 13.79055 & $0.001 *$ \\
\hline Follow up 3m. & $\begin{array}{l}\text { ACL injury < 3 months } \\
\text { ACL injury > 3 months }\end{array}$ & 60 & 14.1833 & 4.72459 & 3.09633 \\
\hline Follow up 6m. & $\begin{array}{l}\text { ACL injury < 3 months } \\
\text { ACL injury > 3 months }\end{array}$ & 60 & 9.6500 & 2.19784 \\
\hline Follow up 12m. & $\begin{array}{l}\text { ACL injury < 3 months } \\
\text { ACL injury > 3 months }\end{array}$ & 60 & 6.5000 & 0 \\
\hline
\end{tabular}

Table (3): Comparison between both groups using the International Knee Documentation Committee (IKDC) scale; sport activity q $(8)$.

\begin{tabular}{|c|c|c|c|c|c|}
\hline Sport activity q(8) & Group case & N. & Mean & Std. Deviation & p.value \\
\hline \multirow{2}{*}{ Pre-operative } & ACL injury < 3 months & 30 & 3.5333 & .81931 & .182 \\
& ACL injury > 3 months & 30 & 3.2333 & .89763 & \\
\hline \multirow{2}{*}{ Follow up 3m. } & ACL injury < 3 months & 30 & 1.4000 & .56324 & .079 \\
& ACL injury > 3 months & 30 & 1.7667 & .97143 & \\
\hline \multirow{2}{*}{ Follow up 6m. } & ACL injury < 3 months & 30 & 1.0000 & .00000 & \multirow{2}{*}{.155} \\
& ACL injury > 3 months & 30 & 1.0667 & .25371 & \\
\hline \multirow{2}{*}{ Follow up 12m. } & ACL injury < 3 months & 30 & 1.0000 & $.00000^{\mathrm{a}}$ & \multirow{2}{*}{$-{ }^{\mathrm{m}}$} \\
& ACL injury > 3 months & 30 & 1.0000 & $.00000^{\mathrm{a}}$ & \\
\hline
\end{tabular}


Table (4): Comparison between both groups using the International Knee Documentation Committee (IKDC) scale; sport activity $\mathbf{q}(9)$.

\begin{tabular}{|c|c|c|c|c|c|c|c|c|c|c|c|c|c|c|c|c|c|c|c|c|c|}
\hline \multirow{4}{*}{$\begin{array}{c}\text { Sport } \\
\text { activity q(9) }\end{array}$} & \multicolumn{10}{|c|}{ ACL injury < 3 months } & \multirow{2}{*}{\multicolumn{10}{|c|}{\begin{tabular}{|l|} 
ACL injury > 3 months \\
upset your ability to
\end{tabular}}} & \multirow{4}{*}{$\mathbf{P}$} \\
\hline & \multicolumn{10}{|c|}{ How does your knee upset your ability to } & & & & & & & & & & & \\
\hline & \multicolumn{2}{|c|}{$\begin{array}{c}\text { Not } \\
\text { difficul } \\
\mathbf{t} \text { at all }\end{array}$} & \multicolumn{2}{|c|}{$\begin{array}{c}\text { Minimu } \\
\mathbf{m} \\
\text { Difficult }\end{array}$} & \multicolumn{2}{|c|}{$\begin{array}{c}\text { Modera } \\
\text { tely } \\
\text { Difficul } \\
t\end{array}$} & \multicolumn{2}{|c|}{$\begin{array}{c}\text { Extrem } \\
\text { ely } \\
\text { difficult }\end{array}$} & \multicolumn{2}{|c|}{$\begin{array}{l}\text { Unabl } \\
\text { e } \\
\text { to do }\end{array}$} & \multicolumn{2}{|c|}{$\begin{array}{c}\text { Not } \\
\text { difficul } \\
t \text { at all }\end{array}$} & \multicolumn{2}{|c|}{$\underset{\text { um }}{\operatorname{Minim}}$} & \multicolumn{2}{|c|}{$\begin{array}{c}\text { Moder } \\
\text { ately } \\
\text { Difficul } \\
\mathbf{t}\end{array}$} & \multicolumn{2}{|c|}{$\underset{\mathbf{m}}{\operatorname{Minimu}}$} & \multicolumn{2}{|c|}{$\begin{array}{l}\text { Unable } \\
\text { to do }\end{array}$} & \\
\hline & $\mathbf{N}$. & $\%$ & N. & $\%$ & N. & $\%$ & $\mathbf{N}$. & $\%$ & $\mathbf{N}$ & $\%$ & $\mathbf{N}$. & $\%$ & $\mathbf{N}$. & $\%$ & $\mathbf{N}$. & $\%$ & $\mathbf{N}$. & $\%$ & $\mathbf{N}$. & $\%$ & \\
\hline \multicolumn{22}{|c|}{ Go up steps } \\
\hline $\begin{array}{l}\text { Pre- } \\
\text { operative }\end{array}$ & 1 & $\begin{array}{l}3 . \\
3\end{array}$ & 2 & 6.7 & 19 & $\begin{array}{r}\mathbf{6 3} \\
.3 \\
\end{array}$ & 8 & $\begin{array}{l}26 \\
.7 \\
\end{array}$ & $\mathbf{0}$ & $\begin{array}{l}\mathbf{0} \\
\mathbf{0}\end{array}$ & 7 & $\begin{array}{c}23 . \\
3\end{array}$ & 11 & $\begin{array}{l}36 \\
.7 \\
\end{array}$ & 10 & $\begin{array}{l}\mathbf{3 3} \\
.3 \\
\end{array}$ & 2 & 6.7 & $\mathbf{0}$ & $\mathbf{0 . 0}$ & $\begin{array}{l}\mathbf{0 . 0 0} \\
1 * *\end{array}$ \\
\hline $\begin{array}{l}\text { After } \\
\text { 3months }\end{array}$ & 10 & $\begin{array}{l}33 \\
.3\end{array}$ & 14 & $\begin{array}{c}46 . \\
7\end{array}$ & 6 & $\begin{array}{l}20 \\
.0\end{array}$ & $\mathbf{0}$ & $\begin{array}{l}\mathbf{0} \\
\mathbf{0}\end{array}$ & $\mathbf{0}$ & $\begin{array}{l}\mathbf{0} \\
\mathbf{0}\end{array}$ & 6 & $\begin{array}{c}20 . \\
0\end{array}$ & 19 & $\begin{array}{l}\mathbf{6 3} \\
.3 \\
\end{array}$ & 5 & $\begin{array}{l}16 \\
.7\end{array}$ & $\mathbf{0}$ & $\mathbf{0 . 0}$ & $\mathbf{0}$ & $\mathbf{0 . 0}$ & $\mathbf{0 . 3 9}$ \\
\hline $\begin{array}{l}\text { After } \\
\text { 6months }\end{array}$ & 30 & $\begin{array}{l}\mathbf{1 0} \\
\mathbf{0}\end{array}$ & $\mathbf{0}$ & $\mathbf{0 . 0}$ & $\mathbf{0}$ & $\mathbf{0}$ & $\mathbf{0}$ & $\mathbf{0 .}$ & $\mathbf{0}$ & $\mathbf{0 .}$ & 27 & $\begin{array}{c}90 . \\
0\end{array}$ & 1 & $\begin{array}{l}3 . \\
3\end{array}$ & 2 & $\begin{array}{l}6 . \\
7 \\
\end{array}$ & $\mathbf{0}$ & 0.0 & $\mathbf{0}$ & $\mathbf{0 . 0}$ & 0.26 \\
\hline $\begin{array}{l}\text { After12mont } \\
\text { hs }\end{array}$ & 30 & $\begin{array}{c}10 \\
0\end{array}$ & $\mathbf{0}$ & $\mathbf{0 . 0}$ & $\mathbf{0}$ & $\mathbf{0}$ & $\mathbf{0}$ & $\begin{array}{l}\mathbf{0} \\
\mathbf{0}\end{array}$ & $\mathbf{0}$ & $\mathbf{0 .}$ & 30 & $\begin{array}{c}10 \\
0\end{array}$ & $\mathbf{0}$ & $\begin{array}{l}\mathbf{0} \\
\mathbf{0}\end{array}$ & $\mathbf{0}$ & $\begin{array}{l}\mathbf{0} \\
\mathbf{0}\end{array}$ & $\mathbf{0}$ & 0.0 & $\mathbf{0}$ & $\mathbf{0 . 0}$ & $\cdots$ \\
\hline \multicolumn{22}{|c|}{ Go down steps } \\
\hline $\begin{array}{l}\text { Pre- } \\
\text { operative }\end{array}$ & 1 & $\begin{array}{l}3 . \\
33\end{array}$ & 2 & 6.7 & 20 & $\begin{array}{l}66 \\
.7\end{array}$ & 7 & $\begin{array}{l}23 \\
.3\end{array}$ & $\mathbf{0}$ & $\begin{array}{l}\mathbf{0} \\
\mathbf{0}\end{array}$ & 6 & $\begin{array}{l}20 \\
.0\end{array}$ & 13 & $\begin{array}{r}43 \\
.3\end{array}$ & 6 & $\begin{array}{l}20 \\
.0\end{array}$ & 5 & $\begin{array}{c}16 . \\
7\end{array}$ & $\mathbf{0}$ & $\mathbf{0 . 0}$ & $\begin{array}{l}0.00 \\
1 * *\end{array}$ \\
\hline $\begin{array}{l}\text { After } \\
\text { 3months }\end{array}$ & 10 & $\begin{array}{l}33 \\
.3\end{array}$ & 14 & $\begin{array}{c}46 . \\
7\end{array}$ & 6 & $\begin{array}{l}20 \\
.0\end{array}$ & $\mathbf{0}$ & $\begin{array}{l}0 . \\
\mathbf{0}\end{array}$ & $\mathbf{0}$ & $\begin{array}{l}\text { 0. } \\
\text { 0 }\end{array}$ & 8 & $\begin{array}{r}26 \\
.7\end{array}$ & 17 & $\begin{array}{l}56 \\
.7\end{array}$ & 5 & $\begin{array}{l}16 \\
.7\end{array}$ & $\mathbf{0}$ & $\mathbf{0 . 0}$ & $\mathbf{0}$ & $\mathbf{0 . 0}$ & 0.74 \\
\hline $\begin{array}{l}\text { After } \\
\text { 6months }\end{array}$ & 30 & $\begin{array}{c}10 \\
\mathbf{0}\end{array}$ & $\mathbf{0}$ & $\mathbf{0 . 0}$ & $\mathbf{0}$ & $\begin{array}{l}\mathbf{0} \\
\mathbf{0}\end{array}$ & $\mathbf{0}$ & $\begin{array}{l}0 . \\
\mathbf{0}\end{array}$ & $\mathbf{0}$ & $\begin{array}{l}0 . \\
\text { 0 }\end{array}$ & 26 & $\begin{array}{l}86 \\
.7\end{array}$ & 2 & $\begin{array}{l}6 . \\
7\end{array}$ & 2 & $\begin{array}{l}6 . \\
7\end{array}$ & $\mathbf{0}$ & $\mathbf{0 . 0}$ & $\mathbf{0}$ & $\mathbf{0 . 0}$ & 0.11 \\
\hline $\begin{array}{l}\text { After12mont } \\
\text { hs }\end{array}$ & 30 & $\begin{array}{c}10 \\
0\end{array}$ & $\mathbf{0}$ & $\mathbf{0 . 0}$ & $\mathbf{0}$ & $\begin{array}{l}\mathbf{0} \\
\mathbf{0}\end{array}$ & $\mathbf{0}$ & $\begin{array}{l}\mathbf{0} \\
\mathbf{0}\end{array}$ & $\mathbf{0}$ & $\begin{array}{l}0 . \\
\mathbf{0}\end{array}$ & 30 & $\begin{array}{c}10 \\
0\end{array}$ & $\mathbf{0}$ & $\mathbf{0}$ & $\mathbf{0}$ & $\begin{array}{l}\mathbf{0} \\
\mathbf{0}\end{array}$ & $\mathbf{0}$ & $\mathbf{0 . 0}$ & $\mathbf{0}$ & $\mathbf{0 . 0}$ & -- \\
\hline \multicolumn{22}{|c|}{ Go down on your knees on the front of your knee } \\
\hline $\begin{array}{l}\begin{array}{l}\text { Pre- } \\
\text { operative }\end{array} \\
\end{array}$ & $\mathbf{0}$ & $\begin{array}{l}\mathbf{0} \\
\mathbf{0}\end{array}$ & 14 & $\begin{array}{c}46 . \\
7 \\
\end{array}$ & 10 & $\begin{array}{l}33 \\
.3 \\
\end{array}$ & 6 & $\begin{array}{l}20 \\
.0 \\
\end{array}$ & $\mathbf{0}$ & $\begin{array}{l}\mathbf{0} \\
\mathbf{0}\end{array}$ & 4 & $\begin{array}{r}13 \\
.3 \\
\end{array}$ & 15 & $\begin{array}{l}\mathbf{5 0} \\
.0 \\
\end{array}$ & 6 & $\begin{array}{l}20 \\
.0 \\
\end{array}$ & 4 & $\begin{array}{c}46 . \\
7 \\
\end{array}$ & 1 & 3.3 & 0.16 \\
\hline $\begin{array}{l}\text { After3month } \\
\mathrm{s}\end{array}$ & 15 & $\begin{array}{l}\mathbf{5 0} \\
.0 \\
\end{array}$ & 14 & $\begin{array}{c}46 . \\
7 \\
\end{array}$ & 1 & $\begin{array}{l}3 . \\
3 \\
\end{array}$ & $\mathbf{0}$ & $\begin{array}{l}0 . \\
\mathbf{0}\end{array}$ & $\mathbf{0}$ & $\begin{array}{l}\mathbf{0} \\
\mathbf{0}\end{array}$ & 14 & $\begin{array}{l}46 \\
.7 \\
\end{array}$ & 14 & $\begin{array}{l}46 \\
.7 \\
\end{array}$ & 1 & $\begin{array}{l}3 . \\
3 \\
\end{array}$ & $\mathbf{0}$ & $\mathbf{0 . 0}$ & 1 & 3.3 & 0.79 \\
\hline $\begin{array}{l}\text { After } \\
\text { 6months }\end{array}$ & 29 & $\begin{array}{l}96 \\
.7\end{array}$ & 1 & 3.3 & $\mathbf{0}$ & $\begin{array}{l}\mathbf{0} \\
\mathbf{0}\end{array}$ & $\mathbf{0}$ & $\begin{array}{l}\mathbf{0} \\
\mathbf{0}\end{array}$ & $\mathbf{0}$ & $\mathbf{0}$ & 26 & $\begin{array}{r}86 \\
.7\end{array}$ & 4 & $\begin{array}{l}\mathbf{1 3} \\
.3\end{array}$ & $\mathbf{0}$ & $\begin{array}{l}\mathbf{0} \\
\mathbf{0}\end{array}$ & $\mathbf{0}$ & 0.0 & $\mathbf{0}$ & $\mathbf{0 . 0}$ & $\begin{array}{c}0.17 \\
7\end{array}$ \\
\hline $\begin{array}{l}\text { After12mont } \\
\text { hs }\end{array}$ & 30 & $\begin{array}{c}10 \\
\mathbf{0}\end{array}$ & $\mathbf{0}$ & $\mathbf{0 . 0}$ & $\mathbf{0}$ & $\begin{array}{l}0 . \\
\mathbf{0}\end{array}$ & $\mathbf{0}$ & $\begin{array}{l}0 . \\
\mathbf{0}\end{array}$ & $\mathbf{0}$ & $\begin{array}{l}0 . \\
\mathbf{0}\end{array}$ & 30 & $\begin{array}{c}10 \\
0\end{array}$ & $\mathbf{0}$ & $\mathbf{0}$ & 0 & $\begin{array}{l}0 . \\
\mathbf{0}\end{array}$ & $\mathbf{0}$ & 0.0 & $\mathbf{0}$ & $\mathbf{0 . 0}$ & ---- \\
\hline \multicolumn{22}{|c|}{ Dumpy } \\
\hline $\begin{array}{l}\text { Pre- } \\
\text { operative }\end{array}$ & $\mathbf{0}$ & $\begin{array}{l}\mathbf{0} \\
\mathbf{0}\end{array}$ & 8 & $\begin{array}{c}26 . \\
7\end{array}$ & 8 & $\begin{array}{l}26 \\
.7 \\
\end{array}$ & 10 & $\begin{array}{l}33 \\
.3 \\
\end{array}$ & 4 & $\begin{array}{l}13 \\
.3 \\
\end{array}$ & 1 & $\begin{array}{l}3 . \\
3\end{array}$ & 9 & $\begin{array}{c}30 \\
.0 \\
\end{array}$ & 11 & $\begin{array}{l}36 \\
.7 \\
\end{array}$ & 5 & $\begin{array}{c}16 . \\
7\end{array}$ & 4 & $\begin{array}{c}13 . \\
3\end{array}$ & .525 \\
\hline $\begin{array}{l}\text { After } \\
\text { 3months }\end{array}$ & 16 & $\begin{array}{l}53 \\
.3 \\
\end{array}$ & $\begin{array}{l}1 \\
3 \\
\end{array}$ & $\begin{array}{c}43 . \\
3\end{array}$ & 1 & $\begin{array}{l}3 . \\
3\end{array}$ & $\mathbf{0}$ & $\begin{array}{l}0 . \\
\mathbf{0}\end{array}$ & $\mathbf{0}$ & $\begin{array}{l}0 . \\
\mathbf{0}\end{array}$ & 5 & \begin{tabular}{|l|}
16 \\
.7 \\
\end{tabular} & 18 & $\begin{array}{l}60 \\
.0 \\
\end{array}$ & 4 & $\begin{array}{l}13 \\
.3 \\
\end{array}$ & 1 & 3.3 & 2 & 6.7 & $\begin{array}{c}\mathbf{0 . 0 2} \\
* \\
\end{array}$ \\
\hline $\begin{array}{l}\text { After } \\
\text { 6months } \\
\end{array}$ & 29 & $\begin{array}{l}96 \\
.7 \\
\end{array}$ & 1 & 3.3 & $\mathbf{0}$ & $\mathbf{0}$ & $\mathbf{0}$ & $\begin{array}{l}\mathbf{0} \\
\mathbf{0}\end{array}$ & $\mathbf{0}$ & $\mathbf{0}$ & 24 & $\begin{array}{c}80 \\
.0 \\
\end{array}$ & 5 & $\begin{array}{l}16 \\
.7 \\
\end{array}$ & $\mathbf{0}$ & $\begin{array}{l}\mathbf{0} \\
\mathbf{0}\end{array}$ & 1 & 3.3 & $\mathbf{0}$ & $\mathbf{0 . 0}$ & 0.12 \\
\hline $\begin{array}{l}\text { After12mont } \\
\text { hs }\end{array}$ & 30 & $\begin{array}{c}10 \\
\mathbf{0}\end{array}$ & $\mathbf{0}$ & $\mathbf{0}$ & $\mathbf{0}$ & $\begin{array}{l}\mathbf{0 .} \\
\mathbf{0}\end{array}$ & $\mathbf{0}$ & $\begin{array}{l}\mathbf{0} \\
\mathbf{0}\end{array}$ & $\mathbf{0}$ & $\begin{array}{l}\mathbf{0} \\
\mathbf{0}\end{array}$ & 30 & $\begin{array}{c}10 \\
0 \\
\end{array}$ & $\mathbf{0}$ & $\mathbf{0}$ & $\mathbf{0}$ & $\begin{array}{l}\mathbf{0} \\
\mathbf{0}\end{array}$ & $\mathbf{0}$ & 0.0 & $\mathbf{0}$ & $\mathbf{0 . 0}$ & --- \\
\hline \multicolumn{22}{|c|}{ Be seated with your knee bent } \\
\hline $\begin{array}{c}\text { Pre- } \\
\text { operative }\end{array}$ & $\mathbf{0}$ & $\begin{array}{l}\mathbf{0} \\
\mathbf{0}\end{array}$ & $\begin{array}{l}1 \\
2\end{array}$ & $\begin{array}{c}40 . \\
0\end{array}$ & 9 & $\begin{array}{c}30 \\
.0\end{array}$ & 8 & $\begin{array}{l}26 \\
.7\end{array}$ & 1 & $\begin{array}{l}3 . \\
3\end{array}$ & $\mathbf{0}$ & $\begin{array}{l}0 . \\
\text { 0 }\end{array}$ & 13 & $\begin{array}{l}43 \\
.3\end{array}$ & 9 & $\begin{array}{c}30 \\
.0\end{array}$ & 5 & $\begin{array}{c}16 . \\
7\end{array}$ & 3 & $\begin{array}{c}10 . \\
0\end{array}$ & 0.63 \\
\hline $\begin{array}{c}\text { After } \\
\text { 3months }\end{array}$ & 15 & $\begin{array}{l}50 \\
.0 \\
\end{array}$ & $\begin{array}{l}1 \\
5\end{array}$ & $\begin{array}{c}50 . \\
0\end{array}$ & $\mathbf{0}$ & $\begin{array}{l}\mathbf{0 .} \\
\mathbf{0}\end{array}$ & $\mathbf{0}$ & $\begin{array}{l}0 . \\
\mathbf{0}\end{array}$ & $\mathbf{0}$ & $\begin{array}{l}\mathbf{0} \\
\mathbf{0}\end{array}$ & 7 & \begin{tabular}{|l|}
23 \\
.3 \\
\end{tabular} & 15 & $\begin{array}{l}50 \\
.0 \\
\end{array}$ & 5 & $\begin{array}{l}16 \\
.7 \\
\end{array}$ & 1 & 3.3 & 2 & 6.7 & $\begin{array}{c}\mathbf{0 . 0 2} \\
* \\
\end{array}$ \\
\hline $\begin{array}{c}\text { After } \\
\text { 6months }\end{array}$ & 29 & $\begin{array}{l}96 \\
.7 \\
\end{array}$ & 1 & 3.3 & $\mathbf{0 .}$ & $\mathbf{0}$ & $\mathbf{0}$ & $\begin{array}{l}0 . \\
\mathbf{0}\end{array}$ & $\mathbf{0}$ & $\mathbf{0}$ & 27 & $\begin{array}{c}90 \\
.0 \\
\end{array}$ & 2 & $\begin{array}{l}6 . \\
7 \\
\end{array}$ & $\mathbf{0}$ & $\begin{array}{l}\mathbf{0 .} \\
\mathbf{0}\end{array}$ & 1 & 3.3 & $\mathbf{0}$ & $\mathbf{0 . 0}$ & 0.49 \\
\hline $\begin{array}{c}\text { After12mont } \\
\text { hs } \\
\end{array}$ & 30 & $\begin{array}{c}10 \\
\mathbf{0}\end{array}$ & $\mathbf{0}$ & 00. & $\mathbf{0}$ & $\begin{array}{l}\text { 0. } \\
\text { 0 }\end{array}$ & $\mathbf{0}$ & $\begin{array}{l}\mathbf{0} \\
\mathbf{0}\end{array}$ & $\mathbf{0}$ & $\begin{array}{l}\mathbf{0} \\
\mathbf{0}\end{array}$ & 30 & $\begin{array}{c}10 \\
\mathbf{0}\end{array}$ & $\mathbf{0}$ & $\begin{array}{l}\mathbf{0} \\
\mathbf{0}\end{array}$ & $\mathbf{0}$ & $\begin{array}{l}\text { 0. } \\
\mathbf{0}\end{array}$ & $\mathbf{0}$ & 0.0 & $\mathbf{0}$ & $\mathbf{0 . 0}$ & ---- \\
\hline \multicolumn{22}{|c|}{ Upswing from a chair } \\
\hline $\begin{array}{c}\text { Pre- } \\
\text { operative }\end{array}$ & $\mathbf{0}$ & $\begin{array}{l}\mathbf{0} \\
\mathbf{0}\end{array}$ & $\begin{array}{l}1 \\
7 \\
\end{array}$ & $\begin{array}{c}56 . \\
7 \\
\end{array}$ & 11 & $\begin{array}{l}36 \\
.7 \\
\end{array}$ & 1 & $\begin{array}{l}3 . \\
3 \\
\end{array}$ & 1 & $\begin{array}{l}3 . \\
3 \\
\end{array}$ & 6 & $\begin{array}{c}20 \\
.0 \\
\end{array}$ & 13 & $\begin{array}{l}43 \\
.3 \\
\end{array}$ & 8 & $\begin{array}{l}26 \\
.7 \\
\end{array}$ & 3 & $\begin{array}{c}10 . \\
0\end{array}$ & $\mathbf{0}$ & $\mathbf{0 . 0}$ & 0.06 \\
\hline $\begin{array}{c}\text { After } \\
\text { 3months }\end{array}$ & 12 & $\begin{array}{l}40 \\
.0\end{array}$ & $\begin{array}{l}1 \\
7\end{array}$ & $\begin{array}{c}56 . \\
7\end{array}$ & 1 & $\begin{array}{l}3 . \\
3\end{array}$ & $\mathbf{0}$ & $\begin{array}{l}\mathbf{0} \\
\mathbf{0}\end{array}$ & $\mathbf{0}$ & $\begin{array}{l}\text { 0. } \\
\text { 0 }\end{array}$ & 13 & $\begin{array}{c}43 \\
.3\end{array}$ & 10 & $\begin{array}{c}\mathbf{3 3} \\
.3\end{array}$ & 5 & $\begin{array}{c}16 \\
.7\end{array}$ & 2 & 6.7 & $\mathbf{0}$ & $\mathbf{0 . 0}$ & 0.08 \\
\hline
\end{tabular}




\begin{tabular}{|c|c|c|c|c|c|c|c|c|c|c|c|c|c|c|c|c|c|c|c|c|c|}
\hline \multirow{4}{*}{$\begin{array}{c}\text { Sport } \\
\text { activity q(9) }\end{array}$} & \multicolumn{10}{|c|}{ ACL injury < 3 months } & \multicolumn{10}{|c|}{ ACL injury > 3 months } & \multirow{4}{*}{$\mathbf{P}$} \\
\hline & \multicolumn{20}{|c|}{ How does your knee upset your ability to } & \\
\hline & \multicolumn{2}{|c|}{$\begin{array}{c}\text { Not } \\
\text { difficul } \\
t \text { at all }\end{array}$} & \multicolumn{2}{|c|}{$\begin{array}{c}\text { Minimu } \\
\text { Difficult }\end{array}$} & \multicolumn{2}{|c|}{$\begin{array}{c}\text { Modera } \\
\text { tely } \\
\text { Difficul } \\
t \\
\end{array}$} & \multicolumn{2}{|c|}{$\begin{array}{c}\text { Extrem } \\
\text { ely } \\
\text { difficult }\end{array}$} & \multicolumn{2}{|c|}{$\begin{array}{c}\text { Unabl } \\
\text { e } \\
\text { to do }\end{array}$} & \multicolumn{2}{|c|}{$\begin{array}{c}\text { Not } \\
\text { difficul } \\
\text { t at all }\end{array}$} & \multicolumn{2}{|c|}{$\underset{\text { um }}{\operatorname{Minim}}$} & \multicolumn{2}{|c|}{$\begin{array}{c}\text { Moder } \\
\text { ately } \\
\text { Difficul } \\
t \\
\end{array}$} & \multicolumn{2}{|c|}{$\underset{\mathbf{m}}{\operatorname{Minimu}}$} & \multicolumn{2}{|c|}{$\begin{array}{l}\text { Unable } \\
\text { to do }\end{array}$} & \\
\hline & $\mathbf{N}$. & $\%$ & N. & $\%$ & $\mathbf{N}$. & $\%$ & $\mathbf{N}$. & $\%$ & $\mathbf{N}$ & $\%$ & N. & $\%$ & $\mathbf{N}$. & $\%$ & $\mathbf{N}$. & $\%$ & N. & $\%$ & N. & $\%$ & \\
\hline $\begin{array}{c}\text { After } \\
\text { 6months }\end{array}$ & 29 & $\begin{array}{c}96 \\
.7\end{array}$ & 1 & 3.3 & $\mathbf{0 .}$ & $\mathbf{0}$ & $\mathbf{0}$ & $\begin{array}{l}0 . \\
0\end{array}$ & $\mathbf{0}$ & $\mathbf{0}$ & 23 & $\begin{array}{l}76 \\
.7\end{array}$ & 5 & $\begin{array}{l}16 \\
.7\end{array}$ & 2 & $\begin{array}{l}6 . \\
7\end{array}$ & $\mathbf{0}$ & 0.0 & $\mathbf{0}$ & 0.0 & 0.06 \\
\hline $\begin{array}{c}\text { After12mont } \\
\text { hs }\end{array}$ & 30 & $\begin{array}{c}10 \\
\mathbf{0}\end{array}$ & $\mathbf{0}$ & 0.0 & $\mathbf{0}$ & $\begin{array}{l}\mathbf{0} \\
\mathbf{0}\end{array}$ & $\mathbf{0}$ & $\begin{array}{l}\mathbf{0} \\
\mathbf{0}\end{array}$ & $\mathbf{0}$ & $\begin{array}{l}\mathbf{0} \\
\mathbf{0}\end{array}$ & 30 & $\begin{array}{c}10 \\
\mathbf{0}\end{array}$ & $\mathbf{0}$ & $\mathbf{0}$ & $\mathbf{0}$ & $\begin{array}{l}\mathbf{0} \\
\mathbf{0}\end{array}$ & $\mathbf{0}$ & 0.0 & $\mathbf{0}$ & 0.0 & ---- \\
\hline \multicolumn{22}{|c|}{ Track straight ahead } \\
\hline $\begin{array}{c}\text { Pre- } \\
\text { operative }\end{array}$ & $\mathbf{0}$ & $\mathbf{0}$ & $\mathbf{0}$ & 0.0 & 5 & $\begin{array}{l}16 \\
.7 \\
\end{array}$ & 22 & $\begin{array}{l}73 \\
.3 \\
\end{array}$ & 3 & $\begin{array}{l}\mathbf{1 0} \\
.0 \\
\end{array}$ & 2 & $\begin{array}{l}6 . \\
7 \\
\end{array}$ & 4 & $\begin{array}{l}13 \\
.3 \\
\end{array}$ & 2 & $\begin{array}{l}6 . \\
7 \\
\end{array}$ & 17 & $\begin{array}{c}56 . \\
7 \\
\end{array}$ & 5 & $\begin{array}{c}16 . \\
7 \\
\end{array}$ & 0.07 \\
\hline $\begin{array}{c}\text { After } \\
\text { 3months }\end{array}$ & $\mathbf{0}$ & $\mathbf{0}$ & 6 & 20. & 19 & $\begin{array}{l}63 \\
.3 \\
\end{array}$ & 4 & $\begin{array}{r}13 \\
.3 \\
\end{array}$ & 1 & $\begin{array}{l}3 . \\
3\end{array}$ & $\mathbf{0}$ & $\begin{array}{l}\text { 0. } \\
\mathbf{0}\end{array}$ & 5 & $\begin{array}{l}16 \\
.7 \\
\end{array}$ & 41 & $\begin{array}{l}46 \\
.7 \\
\end{array}$ & 9 & $\begin{array}{c}30 . \\
\text { 0 }\end{array}$ & 2 & 6.7 & 0.37 \\
\hline $\begin{array}{c}\text { After } \\
\text { 6months }\end{array}$ & 15 & $\begin{array}{l}\mathbf{5 0} \\
.0\end{array}$ & $\begin{array}{l}1 \\
5\end{array}$ & $\begin{array}{c}50 . \\
0\end{array}$ & $\mathbf{0}$ & $\begin{array}{l}\mathbf{0} \\
\mathbf{0}\end{array}$ & $\mathbf{0}$ & $\begin{array}{l}\mathbf{0} \\
\mathbf{0}\end{array}$ & $\mathbf{0}$ & $\begin{array}{l}. \\
\text { 0 }\end{array}$ & 3 & $\begin{array}{l}10 \\
.0 \\
\end{array}$ & 19 & $\begin{array}{l}63 \\
.3 \\
\end{array}$ & 8 & $\begin{array}{l}26 \\
.7 \\
\end{array}$ & $\mathbf{0}$ & 0.0 & $\mathbf{0}$ & 0.0 & $\begin{array}{l}0.00 \\
1 * *\end{array}$ \\
\hline $\begin{array}{c}\text { After12mont } \\
\text { hs }\end{array}$ & 30 & $\begin{array}{c}10 \\
0\end{array}$ & $\mathbf{0}$ & 0.0 & $\mathbf{0}$ & $\begin{array}{l}0 . \\
0\end{array}$ & $\mathbf{0}$ & $\begin{array}{l}0 . \\
0\end{array}$ & $\mathbf{0}$ & $\begin{array}{l}0 . \\
0\end{array}$ & 30 & $\begin{array}{c}10 \\
\mathbf{0}\end{array}$ & $\mathbf{0}$ & $\begin{array}{l}0 . \\
\mathbf{0}\end{array}$ & $\mathbf{0}$ & $\begin{array}{l}0 . \\
0\end{array}$ & $\mathbf{0}$ & 0.0 & $\mathbf{0}$ & 0.0 & ---- \\
\hline \multicolumn{22}{|c|}{ Hurdle and land on your involved leg } \\
\hline $\begin{array}{c}\text { Pre- } \\
\text { operative }\end{array}$ & $\mathbf{0}$ & $\begin{array}{c}\mathbf{0} \\
\mathbf{0}\end{array}$ & 1 & 3.3 & $\mathbf{0}$ & $\begin{array}{l}0 . \\
\mathbf{0}\end{array}$ & 2 & $\begin{array}{l}6 . \\
7\end{array}$ & $\begin{array}{l}2 \\
7 \\
\end{array}$ & $\begin{array}{l}90 \\
.0 \\
\end{array}$ & 2 & $\begin{array}{l}6 . \\
7 \\
\end{array}$ & 2 & $\begin{array}{l}6 . \\
7 \\
\end{array}$ & 2 & $\begin{array}{l}6 . \\
7 \\
\end{array}$ & $\mathbf{0}$ & $\mathbf{0 . 0}$ & 24 & $\begin{array}{c}80 . \\
0\end{array}$ & 0.16 \\
\hline $\begin{array}{c}\text { After } \\
\text { 3months }\end{array}$ & $\mathbf{0}$ & $\begin{array}{l}\mathbf{0 .} \\
\mathbf{0}\end{array}$ & 1 & 3.3 & 3 & $\begin{array}{l}\mathbf{1 0} \\
.0 \\
\end{array}$ & 19 & $\begin{array}{l}63 \\
.3 \\
\end{array}$ & 7 & $\begin{array}{l}23 \\
.3 \\
\end{array}$ & 1 & $\begin{array}{l}3 . \\
3 \\
\end{array}$ & $\mathbf{0}$ & $\begin{array}{l}\mathbf{0 .} \\
\mathbf{0}\end{array}$ & 5 & $\begin{array}{l}16 \\
.7 \\
\end{array}$ & 12 & $\begin{array}{c}40 . \\
0\end{array}$ & 12 & $\begin{array}{c}40 . \\
0\end{array}$ & 0.24 \\
\hline $\begin{array}{c}\text { After } \\
\text { 6months }\end{array}$ & 7 & $\begin{array}{l}23 \\
.3 \\
\end{array}$ & $\begin{array}{l}1 \\
\mathbf{0}\end{array}$ & $\begin{array}{c}33 . \\
3\end{array}$ & 13 & $\begin{array}{r}43 \\
.3 \\
\end{array}$ & $\mathbf{0}$ & $\begin{array}{l}\mathbf{0} \\
\mathbf{0}\end{array}$ & $\mathbf{0}$ & $\begin{array}{l}\mathbf{0} \\
\mathbf{0}\end{array}$ & $\mathbf{0}$ & $\begin{array}{l}\mathbf{0} \\
\mathbf{0}\end{array}$ & 5 & $\begin{array}{l}16 \\
.7 \\
\end{array}$ & 23 & $\begin{array}{l}76 \\
.7 \\
\end{array}$ & 2 & 6.7 & $\mathbf{0}$ & 0.0 & $\begin{array}{l}0.00 \\
1 * *\end{array}$ \\
\hline $\begin{array}{c}\text { After12mont } \\
\text { hs }\end{array}$ & 30 & $\begin{array}{c}10 \\
\mathbf{0}\end{array}$ & $\mathbf{0}$ & 0.0 & $\mathbf{0}$ & $\begin{array}{l}\mathbf{0} \\
\mathbf{0}\end{array}$ & $\mathbf{0}$ & $\begin{array}{l}\mathbf{0} \\
\mathbf{0}\end{array}$ & $\mathbf{0}$ & $\begin{array}{l}\mathbf{0} \\
\mathbf{0}\end{array}$ & 30 & $\begin{array}{c}10 \\
\mathbf{0}\end{array}$ & $\mathbf{0}$ & 0. & $\mathbf{0}$ & $\begin{array}{l}0 . \\
\mathbf{0}\end{array}$ & $\mathbf{0}$ & $\mathbf{0 . 0}$ & $\mathbf{0}$ & 0.0 & ---- \\
\hline \multicolumn{22}{|c|}{ Stop and start rapidly } \\
\hline $\begin{array}{c}\text { Pre- } \\
\text { operative }\end{array}$ & 3 & $\begin{array}{l}10 \\
.0\end{array}$ & $\begin{array}{l}2 \\
2\end{array}$ & $\begin{array}{c}73 . \\
3\end{array}$ & 3 & $\begin{array}{l}\mathbf{1 0} \\
.0\end{array}$ & 2 & $\begin{array}{c}6 . \\
7\end{array}$ & $\mathbf{0}$ & $\begin{array}{l}0 . \\
0\end{array}$ & 6 & $\begin{array}{l}20 \\
.0\end{array}$ & 17 & $\begin{array}{l}56 \\
.7\end{array}$ & 5 & $\begin{array}{l}16 \\
.7\end{array}$ & 2 & 6.7 & $\mathbf{0}$ & 0.0 & 0.54 \\
\hline $\begin{array}{c}\text { After } \\
\text { 3months }\end{array}$ & 23 & $\begin{array}{l}76 \\
.7 \\
\end{array}$ & 7 & $\begin{array}{l}23 . \\
3\end{array}$ & $\mathbf{0}$ & $\begin{array}{l}\mathbf{0} \\
\mathbf{0}\end{array}$ & $\mathbf{0}$ & $\begin{array}{l}\mathbf{0} \\
\mathbf{0}\end{array}$ & $\mathbf{0}$ & $\begin{array}{l}\mathbf{0 .} \\
\mathbf{0}\end{array}$ & 24 & $\begin{array}{l}80 \\
.0 \\
\end{array}$ & 5 & $\begin{array}{l}16 \\
.7 \\
\end{array}$ & 1 & $\begin{array}{l}3 . \\
3 \\
\end{array}$ & $\mathbf{0}$ & $\mathbf{0 . 0}$ & $\mathbf{0}$ & 0.0 & $\mathbf{0 . 5 0}$ \\
\hline $\begin{array}{c}\text { After } \\
\text { 6months }\end{array}$ & 25 & $\begin{array}{c}83 \\
.3\end{array}$ & 3 & $\begin{array}{c}10 . \\
0\end{array}$ & 2 & $\begin{array}{l}6 . \\
7\end{array}$ & $\mathbf{0}$ & $\begin{array}{l}\mathbf{0} . \\
\mathbf{0}\end{array}$ & $\mathbf{0}$ & $\begin{array}{l}0 . \\
\text { 0 }\end{array}$ & 29 & $\begin{array}{l}96 \\
.7\end{array}$ & 1 & $\begin{array}{l}3 . \\
3\end{array}$ & 0. & $\mathbf{0}$ & $\mathbf{0}$ & $\mathbf{0 . 0}$ & $\mathbf{0}$ & $\mathbf{0}$ & 0.19 \\
\hline $\begin{array}{c}\text { After12mont } \\
\text { hs }\end{array}$ & 30 & $\begin{array}{c}10 \\
0\end{array}$ & $\mathbf{0}$ & $\mathbf{0 . 0}$ & $\mathbf{0}$ & $\begin{array}{l}\text { 0. } \\
\text { 0 }\end{array}$ & $\mathbf{0}$ & $\begin{array}{l}\mathbf{0} \\
\mathbf{0}\end{array}$ & $\mathbf{0}$ & $\begin{array}{l}\text { 0. } \\
\text { 0 }\end{array}$ & 30 & $\begin{array}{c}\mathbf{1 0} \\
\mathbf{0}\end{array}$ & $\mathbf{0}$ & 0. & $\mathbf{0}$ & $\begin{array}{l}\text { 0. } \\
\text { 0 }\end{array}$ & $\mathbf{0}$ & $\mathbf{0 . 0}$ & $\mathbf{0}$ & 0.0 & ---- \\
\hline
\end{tabular}

Table (5):- Comparison between both groups using the International Knee Documentation Committee (IKDC) scale; sport activity q(10).

\begin{tabular}{|c|c|c|c|c|c|}
\hline \multirow{3}{*}{ Variable } & $\begin{array}{c}\text { ACL injury }<3 \\
\text { months }\end{array}$ & $\begin{array}{c}\text { ACL injury }>3 \\
\text { months }\end{array}$ & \multirow{3}{*}{$\mathbf{P 1}$} & \multirow{3}{*}{$\mathbf{P 2}$} & \multirow{3}{*}{$\mathbf{P 3}$} \\
\hline & \multicolumn{2}{|c|}{ Purpose prior to the knee injury } & & & \\
\hline & Mean \pm SD & Mean \pm SD & & & \\
\hline Pre-operative & $4.333 \pm 1.728$ & $4.200 \pm 1.297$ & \multirow{4}{*}{$0.001 *$} & \multirow{4}{*}{$0.001 *$} & .737 \\
\hline Follow up3mo. & $7.966 \pm 1.272$ & $6.233 \pm 1.406$ & & & $0.001^{*}$ \\
\hline Follow up6mo. & $8.600 \pm .855$ & $7.800 \pm .996$ & & & $0.001 *$ \\
\hline Follow up12mo. & $8.0000 \pm .000$ & $8.0000 \pm .000$ & & & ------- \\
\hline Total & $7.225 \pm 2.043$ & $6.558 \pm 1.864$ & & $0.009 *$ & \\
\hline
\end{tabular}

Comparison between: $\quad$ Pl. ACL injury $<3$ months.

P3. ACL injury $>3$ months and ACL injury $<3$ months

P2. ACL injury > 3 months.

Table (1): the mean age of was (24.75 \pm 5.559$)$, regarding level of education; $(70 \%)$ of patients in both groups were highly educated and the highest percent of them $(61.7 \%)$ were students. Table (2): there was a highly statistically significant difference between both groups regarding their symptoms on the IKDC scale P. (0.001**).

Table (3): there was no statistically significant difference between both groups regarding their sporting activity on the IKDC scale. 
Table (4), there was a highly statistically significant difference between patients with an ACL injury $<3$ months and patients with an ACL injury > 3 months regarding how does their knee affect their ability to; go upstairs pre-operatively P.V( $\left.0.001^{*}\right)$, go down stairs pre-operatively $\mathrm{P} . \mathrm{V}\left(0.001^{*}\right)$, squat at 3 months post operatively $\mathrm{P} . \mathrm{V}\left(0.02^{*}\right)$, sit with knees bent at 3 months post operatively $\mathrm{P} . \mathrm{V}(0.02 *)$, run straight ahead at 6 months post operatively P.V(0.001*), and their ability to jump and land on the affected leg at 6 months post operatively P.V(0.002*).

Table (5), there was a highly statistically significant difference regarding mean $\pm \mathrm{SD}$ of the (IKDC) scale; function prior to the knee injury among patients whose ACL injury < 3 months and patients with ACL injury > 3 months, while regarding comparison between both groups there was a statistically significant difference only at 3 and 6 months of follow up.

\section{Discussion}

The mean age were (24.7) years of patients in both groups ACL injury is more common in this age group because of their more active lifestyle as well as higher participation in sports. This result agree with (Mohammed, 2016) who found that ACL is most prevalent in patients with a mean age of (28.2) years. While the result of this study disagrees with (Hussien, 2013) who found that the mean age of patients was 47 years and also disagree with (Collins et al., 2013) who reported that the age of participants ranged from 16 to 52 years.

The majority of patients in both groups were highly educated. This result agrees with (Mohammed, 2016) who found that the majority of patients were highly educated, and disagrees with the study results of (Salem et al., 2012) which showed that the majority of patients were secondary educated.

Regarding occupations, the majority of patients were students. This result disagrees with (Salem et al., 2012) who found that patients were about equally to have a manual or sedentary occupation.

There was a highly statistically significant difference between both groups regarding their symptoms on the IKDC scale pre-operatively and all of the follow up periods, this result disagree with (Marcacci et al., 2013) who reported that there was no significant difference between the groups for International Knee Documentation Committee rating score.

There was no statistically significant difference between both groups regarding their sporting activity on the IKDC scale in relation to the highest level of activity in which they can participate in on a regular basis pre-operatively and all of the follow up periods. There was a highly statistically significant difference between patients with an ACL injury $<3$ months and patients with an ACL injury > 3 months regarding their sporting activity on the IKDC scale regarding how does their knees affect their ability to: go upstairs pre-operatively, go down stairs preoperative, squat at 3 months post operatively, sit with knees bent at 3 months post operatively, run straight ahead at 6 months post operatively and their ability to jump and land on the affected leg at 6 months post operatively. (Bruce \& Robert, 2010) in their study which was conducted on 20 cases at 6 months post operatively found that 13 cases had no problems with squatting $(65 \%), 5$ cases with slightly impaired squatting $(25 \%)$ and 2 cases not beyond $90^{\circ}(10 \%)$, (Huang \& Wang, 2011) activities of daily living preoperatively including sitting down, rising from the sitting position, squatting, going upstairs and down stairs cannot be performed properly, and patients have limited range of motion in the knee joint .

There was a highly statistically significant difference regarding mean \pm SD of the (IKDC) scale; function prior to the knee injury among patients whose ACL injury < 3 months and patients with an ACL injury > 3 months, while regarding comparison between both groups there was a statistically significant difference only at 3 and 6 months of follow up, this result disagree with (Zsolt, 2010) whose results showed that 6 weeks and 4 months after surgery the differences were statistically significant.

This result contradicts the results by (Julie \& Robert 2013) who declared that the IKDC defined no improvement in function as less than 11.5-point change in score, making it likely that our demonstrated difference of 2.7 points would not be clinically important at all three test speed.

\section{Conclusion}

An improvement was observed at $3 \& 6$ months follow up of patients with an ACL injury of less than 3 months than those patients whose injury more than 3 months.

\section{Recommendations}

- Orientation programs on mass media (TVs \& radio) on importance of early medical consultation and early surgery to improve their functional level.

- Providing university students with instructions for seeking medical advice for their sports injuries is of great importance for early diagnosis and management of their injuries.

\section{References:}

1. Aglietti, P., Giron, F., Buzzi, R., Biddau, F., \& Sasso, F., (2014): Anterior cruciate ligament reconstruction: bone-patellar tendon-bone 
compared with double semitendinosus and gracilis tendon grafts. A prospective, randomized clinical trial. J Bone Joint Surg Am. Oct; Vol. (86), Pp. (43-45).

2. Anderson, A., Bergfeld, J., Boland, A., Dye, S., Feagin, J., \& Harner, C., (2000): International Knee Documentation Committee (IKDC) Scale. http://www.orthopaedicscores.com.2-12-2017

3. Baum, E., \& Irrgang, J., (2013): Postoperative anterior cruciate ligament rehabilitation. Controversies in orthopaedic sports medicine. Champaign, IL: Human Kinetics; Pp. (195-200).

4. Bruce, D., \& Robert, J., (2010): The science of ACL Rehabilitation. vol.(40), Pp. (9-20).

5. Collins, J., Katz, J., Donnell-Fink, L., Martin, S., \& Losina, E., (2013): Cumulative incidence of ACL reconstruction after ACL injury in adults, role of age, sex, and race, $2^{\text {nd }}$ edition chapter 5,USA: Clinical Orthopedic.

6. Dugan, S., (2015): Sports-related knee injuries: what gives? Am J Phys Med Rehabil., Feb; Vol.(84), Pp. (22-30)

7. Huang, H., \& Wang, G., (2011): The early results of high-flex total knee arthroplasty: a minimum of 2 years of follow-up. The Journal of Arthroplasty, 08, Vol. (20), No. (5), Pp. (674679).

8. Hussien, S., (2013): Effect of Developing Nursing care standards for patient with anterior cruciate ligament surgery at Assiut university hospital, Unpublished Doctor thesis of Medical and Surgical Nursing, Faculty of Nursing, Assiut university.

9. Julie, A., \& Robert, F., (2013): Assessment of Differences between the Modified Cincinnati and International Knee Documentation Committee Patient Outcome Scores, The American Journal of Sports Medicine, Vol. (37), No. (11), P. (65).

10. Marcacci, M., Zaffagnini, S., Iacono, F., Pia Neri, M,. \& Petitto, A., (2013): Early versus late reconstruction for anterior cruciate ligament rupture. Results after five years of follow-up. Am J Sport Med vol.(23), Pp. (690-693).

11. Mohammed, S., (2016): Impact Of Nursing Rehabilitation Program On Outcome Of Patients Undergoing Arthroscopy For Anterior Cruciate Ligament Injury.iosrjournals, Volume 5, No.(2), Pp.( 29-38).

12.Salem, S., Sharaf, S., Mostafa, M., \& AbdElhaliem, M., (2012): Impact of a Designed Nursing Rehabilitation Program on incidence of complication and length of hospital stay After Anterior Cruciate Ligament (ACL) Reconstruction El-Manial University Hospital. Am J Sport Med vol.(18), Pp. (43-45).
13. Sharonl, K., Heitkemper, L., Patricia, S., \& Linda, N., (2007): medical surgical nursing, Role of Nurse in Rehabilitation, 8th ed., Sunders Elsevier, USA 7th ed,ch (15), p(2430).

14. Shea, K., (2015): The American Academy of Orthopaedic Surgeons evidence-based guideline on management of anterior cruciate ligament njuries. Journal of Bone and Joint Surgery, Vol. (97), Pp. (672).

15.Zsolt, K., (2010): Biomechanics of the knee in healthy and anterior cruciate ligament injured athletes during gait, Am J Sport Med Vol. (19), Pp. (580-590). 